\title{
ON THE EXTREME EIGENVALUES OF TOEPLITZ MATRICES
}

\author{
BY \\ SEYMOUR V. PARTER
}

1. Introduction. Beginning with work of Kac, Murdock, and Szegö [3] (see [2] also) several recent works have been concerned with the asymptotic behavior of the extreme eigenvalues of Toeplitz matrices.

Let $f(\theta)$ be a real valued, Lebesgue integrable function defined on $[-\pi, \pi]$. Let $\left\{C_{j}\right\}$ be the Fourier coefficients of $f(\theta)$, i.e.,

$$
f(\theta) \sim \sum C_{j} e^{i j \theta} .
$$

The matrix $T_{n}[f]=\left(C_{s-j}\right), s, j=0,1, \cdots, n$ is called the $n$th finite section of the infinite Toeplitz matrix $\left(C_{s-j}\right)$ associated with the function $f(\theta)$.

We will be concerned with functions $f(\theta)$ satisfying

Condition A. Let $f(\theta)$ be real, continuous and periodic with period $2 \pi$. Let $\min f(\theta)=f(0)=0$ and let $\theta=0$ be the only value of $\theta(\bmod 2 \pi)$ for which this minimum is attained.

Condition $\mathrm{A}(\alpha)$. Let $\alpha$ be a positive real number. Let $k(\alpha)$ be the smallest integer $\geqq \alpha / 2$. Let $f(\theta)$ satisfy Condition A. Let $g(\theta)=[f(\theta)]^{2 k / \alpha}$. Let $g(\theta)$ have $2 k$ continuous derivatives in some neighborhood of $\theta=0$. Finally, let $g^{(2 k)}(0)$ $=\sigma^{2}>0$ be the first nonvanishing derivative of $g(\theta)$ at $\theta=0$.

Note. The $\alpha$ of this work is twice the $\alpha$ we used in [5].

We remark that the conditions $f(0)=0$ and $\min f(\theta)=f(0)$ are not essential; see $[2 ; 3$ or 8$]$.

The case $\alpha=2$ was studied by Kac, Murdock and Szegö [3] who also studied a related problem for integral operators. Widom [8] also studied the case $\alpha=2$ for both the Toeplitz matrices and the related integral operators.

The case $\alpha=4$ was studied by us in [4].

In [9] Widom obtained results in the integral operator case for $0<\alpha \leqq 2$. Moreover, Widom made a correct conjecture for the case of general $\alpha$ in that case. The validity of his general conjecture is proven in [10].

In [5] we studied the Toeplitz matrices for the case where $\alpha$ is an even integer. We showed that if $\lambda_{v, n}$ are the eigenvalues of $T_{n}[f]$ arranged in nondecreasing order, then for fixed $\nu$ as $n \rightarrow \infty$ we have

$$
(n+2)^{\alpha} \lambda_{\nu, n} \rightarrow \frac{\sigma^{2}}{\alpha !} \Lambda_{\nu}
$$

where $\Lambda_{\nu}$ is the $\nu$ th eigenvalue of

Presented to the Society, April 15, 1961; received by the editors February 2, 1961. 


$$
\left[-\left(\frac{d}{d x}\right)^{2}\right]^{\alpha / 2} U-\Lambda U=0
$$$$
0 \leqq x \leqq 1,
$$

while

$$
U^{(j)}(0)=U^{(j)}(1)=0, \quad j=0,1, \cdots, \frac{\alpha}{2}-1 .
$$

The purpose of this paper is to establish the asymptotic behavior of the $\lambda_{v, n}$ for all $\alpha$. Of course, for $\alpha$ an even integer our results will agree with those described above. The answer is essentially the same as that obtained by Widom [10] except that we are operating in the interval $[0,1]$ rather than $[-1,1]$. Specifically we prove

THEOREM. Let $\lambda_{v, n}$ be the eigenvalues of $T_{n}[f]$ arranged in nondecreasing order. Let $f(\theta)$ satisfy condition $A(\alpha)$. Then, for fixed $\nu$ as $n \rightarrow \infty$, we have

$$
(n+2)^{\alpha} \lambda_{\nu, n} \rightarrow\left[\frac{\sigma}{(2 k) !}\right]^{\alpha / 2 k} \Lambda_{\nu}
$$

where $\Lambda \nu$ is the vth eigenvalue (actually the reciprocal of the eigenvalue) of the integral operator $k_{0}(x, y)=2 k(2 x-1,2 y-1)$ on $[0,1]$ with $k(\xi, \eta)$ being the integral operator described by Widom in [10].

The proof is based on the following ideas. As in [5] we interpret the problem as an eigenvalue problem for a finite-difference operator and concern ourselves (at first) only with weak convergence of the eigenfunctions. The critical new facets are Widom's [10] explicit estimates on the Fourier transforms of the eigenfunctions of $k_{0}(x, y)$ and his observation that it suffices to work with the Fourier transforms.

Thus, a large part of this work is concerned with estimates on the Fourier transforms of piecewise-linear functions. These are all collected in §2, separate from the proof of the theorem. The proof of the theorem is presented in $\S 3$.

The author is indebted to Professor Widom for many stimulating and fruitful discussions during the course of this work.

2. Preliminaries. The basic formula which relates the bilinear form generated by $T_{n}[f]$ to Fourier analysis is the following:

Lemma 1. Let $X=\left(x_{j}\right), Y=\left(y_{j}\right), j=1,2, \cdots, n+1$, be any two $(n+1)$ vectors. Let $H(\theta)=\sum x_{j} e^{-i j \theta}, G(\theta)=\sum y_{j} e^{-i j \theta}$. Then

$$
\left(X, T_{n} Y\right)=\frac{1}{2 \pi} \int_{-\pi}^{\pi} f(\theta) H(\theta) \bar{G}(\theta) d \theta,
$$

and 


$$
(X, Y)=\frac{1}{2 \pi} \int_{-\pi}^{\pi} H(\theta) \bar{G}(\theta) d \theta .
$$

Here we use the conventional "inner product" notation,

$$
(X, Y)=\sum x_{j} \bar{y}_{j}
$$

Proof. Direct computation.

Lemma 2 (Weyl-Courant). Let $A$ be an Hermitian matrix of order $n+1$. Let $\mu_{0}, \mu_{1}, \cdots, \mu_{n}$ be the eigenvalues of $A$ arranged in nondecreasing order. The $\mu_{\nu}, \nu \geqq 1$, may be characterized by

$$
\mu_{\nu}=\operatorname{Max}_{Y_{1}, Y_{2}, \cdots, Y_{\nu-1}}\left\{\operatorname{Min}_{x \neq 0} \frac{(X, A X)}{(X, X)}\right\},
$$

where $Y_{1}, Y_{2}, \cdots, Y_{\nu-1}$ is an arbitrary set of $\nu-1$ vectors and $X$ runs over all nonzero vectors satisfying

$$
\left(Y_{j}, X\right)=0, \quad j=1,2, \cdots, \nu-1 .
$$

Furthermore, for $\nu=0$ we have

$$
\mu_{0}=\operatorname{Min}_{x \neq 0} \frac{(X, A X)}{(X, X)} .
$$

Proof. See [1, Chapter 1].

It follows from this result that if $T$ and $U$ are two Hermitian matrices such that $(x, T x) \leqq(x, U x)$ for all vectors $x$, then each eigenvalue of $T$ is less than or equal to the corresponding eigenvalue of $U$. Thus, Lemma 1 implies that if the eigenvalues of $T_{n}[f]$ are denoted by $\lambda_{\nu, n}$ and the eigenvalues of $T_{n}[g]$ by $\mu_{\nu, n}$, the conditions $f(\theta) \leqq g(\theta)$ for all $\theta$ implies $\lambda_{\nu, n} \leqq \mu_{r, n}$ for all $\nu$ and $n$.

Hence (see $[2 ; 3]$ ) it suffices to consider functions $f(\theta)$ of the following two special forms.

CASE 1. $\alpha<2 k(\alpha)$.

$$
f(\theta)=\beta|\theta|^{\alpha}[1+r(\theta)],
$$

where $r(\theta)$ is an even trigonometric polynomial satisfying

$$
r(0)=0
$$

and

$$
0<\rho_{0} \leqq 1+r(\theta) \leqq \rho
$$

for two positive constants $\rho_{0}$ and $\rho_{1}$, while

$$
\beta=\left[\frac{\sigma^{2}}{(2 k) !}\right]^{\alpha / 2 k} \text {. }
$$


CASE 2. $\alpha=2 k(\alpha)$.

$$
f(\theta)=\beta(1-\cos \theta)^{k}[1+r(\theta)],
$$

where $r(\theta)$ satisfies $(2.3 \mathrm{~b})$ and $(2.3 \mathrm{c})$ while

$$
\beta=2^{k} \frac{\sigma^{2}}{\alpha !} .
$$

It will also be useful to have the "dual" of Lemma 2 .

Lemma 3 (PoINCARÉ-RITz). Under the hypothesis of Lemma 2 the $\mu_{\nu}$ may be characterized by

$$
\mu_{\nu}=\operatorname{Min}_{Y_{1}, Y_{2}, \ldots, Y_{\nu}}\left\{\operatorname{Max}_{X \neq 0} \frac{(X, A x)}{(X, X)}\right\},
$$

where $X$ is a nonzero vector in the subspace spanned by the $\nu$ linearly independent vectors $Y_{1}, Y_{2}, \cdots, Y_{v}$.

Proof. The proof follows from a simple modification of the proof of Lemma 2 found in [1].

We now turn to the properties of the eigenfunctions $\{\phi(x)\}$ of the operator $k_{0}(x, y)$.

LEMMA 4. Let $\left\{\phi^{(v)}(x)\right\}$ be the eigenfunctions of the integral operator $k_{0}(x, y)$ while $\left\{\Lambda_{\nu}\right\}$ are the eigenvalues, i.e.,

$$
\phi^{(\nu)}(x)=\Lambda_{\nu} \int_{0}^{1} k_{0}(x, y) \phi^{(v)}(y) d y, \quad 0 \leqq x \leqq 1 .
$$

Let $\left\{\Phi^{(\nu)}(\xi)\right\}$ be the Fourier transforms of the $\phi^{\nu}(x)$. Then

$$
\begin{aligned}
\phi^{(v)}(x) & =0 & \text { for } x \notin[0,1], \\
|\xi|^{\alpha / 2} \Phi^{(v)}(\xi) & \in L_{2}(-\infty, \infty), &
\end{aligned}
$$

if $\alpha<2 k(\alpha)$ there exists a positive $\epsilon$ such that

$$
|\xi|^{\alpha / 2+\epsilon} \Phi^{(v)}(\xi) \in L_{2}(-\infty, \infty) .
$$

Furthermore, if $\psi(x)$ is any function which vanishes outside $[0,1]$ with Fourier transform $\Psi(\xi)$ satisfying

$$
|\xi|^{\alpha / 2} \Psi(\xi) \in L_{2}(-\infty, \infty),
$$

then

$$
\int_{-\infty}^{\infty}|\xi|^{\alpha} \Psi(\xi) \bar{\Phi}(\xi) d \xi=\Lambda_{\nu} \int_{-\infty}^{\infty} \Psi(\xi) \bar{\Phi}(\xi) d \xi
$$


Proof. See [10].

COROLlaRY. If $\alpha=2 k(\alpha)$ then $k_{0}(x, y)$ is the Green's function of the operator described by (1.1) and (1.1a).

Let $D(h)$ be the forward-difference operator with increment $h$, i.e.,

$$
D(h) G(x)=G(x+h)-G(x) .
$$

LemMa 5. Let $\beta$ be a positive real number, not an integer. Let $g(x)$ be a function which vanishes outside $[0,1]$ with Fourier transform $G(\xi)$. Let $|\xi|^{\beta} G(\xi)$ $\in L_{2}(-\infty, \infty)$. Let $j$ be the smallest integer which is $\geqq \beta$. Then

$$
\int_{-\infty}^{\infty}\left|D^{i}(h) g(x)\right|^{2} d x \leqq C h^{2 \beta} .
$$

Proof. The lemma follows from standard arguments on Fourier transforms; see [7].

LEMMA 6. If $\alpha=2 k(\alpha)$ and $\phi(x)$ is an eigenfunction of $k_{0}(x, y)$, then

$$
\phi(x) \in C_{k-1}(-\infty, \infty)
$$

and $\phi(x)$ has infinitely many derivatives except at $x=0$ and $x=1$.

Proof. These are well-known properties of these eigenfunctions which are readily obtained from (1.1) and (1.1a).

LEMma 7. Let $\left\{G^{(j)}(\xi)\right\}$ be a sequence of functions in $L_{2}(-\infty, \infty)$ which converge pointwise to a function $G^{0}(\xi)$, converge uniformly to $G^{0}(\xi)$ in every compact subset, and also satisfy

$$
\begin{gathered}
\left\|G^{(j)}\right\|=1 \\
H^{(j)}(\xi)=|\xi|^{\beta} G^{(j)}(\xi) \in L_{2}(-\infty, \infty),
\end{gathered}
$$

and

$$
\left\|H^{(j)}\right\| \leqq M
$$

Let $\boldsymbol{\gamma}$ be any number $0 \leqq \gamma<\beta$. There is a subsequence $\left\{G^{\left(j^{\prime}\right)}(\xi)\right\}$ which converges strongly to $G^{0}(\xi)$ and $|\xi|^{\gamma} G^{\left(j^{\prime}\right)}(\xi)$ also converges strongly to $|\xi|^{\gamma} G^{0}(\xi)$. Of course, this implies that $|\xi|^{\gamma} G^{0}(\xi) \in L_{2}(-\infty, \infty)$.

Proof. Condition (2.11a) implies the existence of a subsequence $\left\{G^{\left(j^{\prime}\right)}(\xi)\right\}$ which converges weakly to some function, say $H(\xi)$, and $H(\xi) \in L_{2}$. However, by a theorem of Banach and Saks (see $[6, \S 38]$ ) there is a sub-subsequence whose arithmetic means converge strongly to $H(\xi)$. But these arithmetic means must also converge pointwise to $G^{0}(\xi)$. Thus $G^{0}(\xi)=H(\xi)$. We now show that this weakly convergent subsequence converges strongly.

From 2.11c we have 


$$
\int_{-a}^{a}|\xi|^{2 \beta}\left|G^{(j)}\right|^{2} d \xi+|a|^{2 \beta} \int_{|a|<|\xi|}\left|G^{(j)}\right|^{2} d \xi \leqq M^{2},
$$

for every $a>0$. Let $a$ be chosen so that $a^{2 \beta}>M^{2}$. Using (2.11a) we obtain

$$
\int_{-a}^{a}\left|G^{(j)}\right|^{2} d \xi \geqq 1-\frac{M^{2}}{|a|^{2 \beta}} .
$$

However, on $[-a, a]$ the functions $G^{\left(j^{\prime}\right)}(\xi)$ converge uniformly to $G^{0}(\xi)$. Hence

$$
\left\|G^{0}\right\|^{2} \geqq \int_{-a}^{a}\left|G^{0}(\xi)\right|^{2} d \xi \geqq 1-\frac{M^{2}}{|a|^{2 \beta}} .
$$

Since this holds for all $a>M^{1 / \beta}$ we have

$$
\left\|G^{0}(\xi)\right\| \geqq 1 \text {. }
$$

The reverse inequality follows from Fatou's lemma and the strong convergence of the $G^{\left(j^{\prime}\right)}(\xi)$ to $G^{0}(\xi)$ follows (see $[6, \S 37]$ ). The proof of the lemma for $\boldsymbol{\gamma}>\mathbf{0}$ follows from a simple modification of the above argument.

Let us now interpret the eigenvalue problem $T_{n} X=\lambda X$ as an eigenvalue problem for a finite-difference operator. Let $\Delta x=1 /(n+2)$ and let $x_{j}=j \Delta x$, $j=0, \pm 1, \pm 2, \cdots$ be the lattice points on the real axis. For any function $g(x)$, we denote $g\left(x_{j}\right)$ by $g_{j}$.

Let $P_{n}$ be the class of piecewise-linear real functions $h(x)$ determined by their values at $x_{j}$ which satisfy

$$
h_{j}=0 \text { for } j \leqq 0 \text { and } j \geqq n+2 .
$$

We observe that every function $h(x) \in P_{n}$ corresponds to an $(n+1)$-vector $H=\left(h_{j}\right), j=1,2, \cdots, n+1$, and conversely. Let $S_{n}$ be the operator defined on $P_{n}$ by

$$
\left(S_{n} h\right)_{j} \equiv\left\{\begin{array}{cl}
(n+2)^{\alpha} T_{n}[f], & j=1,2, \cdots n+1 \\
0, & \text { otherwise. }
\end{array}\right.
$$

For any two functions $h(x), g(x) \in P_{n}$ we define

$$
[h, g] \equiv \Delta x \sum h_{j} g_{j}=\Delta x(H, G),
$$

where $H$ and $G$ are the $(n+1)$ vectors which correspond to $h$ and $g$ respectively.

Lemma 8. Let $h(x) \in P_{n}$ and let $H(\xi)$ be its Fourier transform, i.e.,

$$
H(\xi) \equiv \int_{-\infty}^{\infty} h(x) e^{i x \xi} d x=\int_{0}^{1} h(x) e^{i x \xi} d x .
$$

Then 


$$
H(\xi)=\frac{2(1-\cos \xi /(n+2))}{(\xi /(n+2))^{2}}\left[\frac{1}{n+2} \sum h_{j} e^{i x j \xi}\right] .
$$

Proof. Direct computation.

Let $g(x)$ be any "smooth" function which vanishes outside $[0,1]$. We wish to obtain a sequence of functions $g^{(n)}(x) \in P_{n}$ which converge to $g(x)$. To this end we define

$$
g_{i}^{(n)} \equiv(n+1) \int_{(j-1) /(n+1)}^{j /(n+1)} g(x) d x .
$$

Lemma 9. Let $g(x)$ and $g^{(n)}(x)$ be two functions related by (2.18). Let $j$ and $r$ be any two integers. Then

$$
\int_{-\infty}^{\infty}\left|D^{i}\left(\frac{r}{n+2}\right) g^{(n)}(x)\right|^{2} d x \leqq \frac{4}{3} \int_{-\infty}^{\infty}\left|D^{i}\left(\frac{r}{n+1}\right) g(x)\right|^{2} d x .
$$

Proof. Consider

$$
I_{s}=\int_{x_{s}}^{x_{s+1}}\left|D^{i}\left(\frac{r}{n+2}\right) g^{(n)}(x)\right|^{2} d x .
$$

It is easy to verify that

$$
\begin{aligned}
I_{8} \leqq 2(n+1)^{2}(n+ & 2)^{2}\left\{\int_{x_{8}}^{x_{8+1}}\left(x-x_{8}\right)^{2} \cdot\left[\int_{(8-1) /(n+1)}^{s /(n+1)} D^{j}\left(\frac{r}{n+1}\right) g(\xi) d \xi\right]^{2} d x\right. \\
& \left.+\int_{x_{8}}^{x_{8+1}}\left(x_{8+1}-x\right)^{2}\left[\int_{8 /(n+1)}^{(8+1) /(n+1)} D^{j}\left(\frac{r}{n+1}\right) g(\xi) d \xi\right]^{2} d x\right\} .
\end{aligned}
$$

Upon applying Schwarz's inequality and integrating, we find

$$
\begin{aligned}
& I_{s} \leqq \frac{2(n+1)}{3(n+2)}\left\{\int_{(s-1) /(n+1)}^{s /(n+1)}\left|D^{j}\left(\frac{r}{n+1}\right) g(\xi)\right|^{2} d \xi\right. \\
&\left.+\int_{s /(n+1)}^{(s+1) /(n+1)}\left|D^{j}\left(\frac{r}{n+1}\right) g(\xi)\right|^{2} d \xi\right\} .
\end{aligned}
$$

The desired conclusion then follows after summing on $s$.

LEMMA 10. Let $g(x)$ be any function which vanishes outside $[0,1]$ with Fourier transform $G(\xi)$. Let $\alpha<2 k(\alpha)$. Let $G(\xi)$ satisfy $|\xi| \alpha / 2+\epsilon G(\xi) \in L_{2}, \epsilon>0$. Let $g^{(n)}(x) \in P_{n}$ be the sequence of functions obtained from $g(x)$ by (2.18). Let $G^{(n)}(\xi)$ be the Fourier transforms of the $g^{(n)}(x)$. There is a constant $C_{0}$ depending only on $g(x)$ such that

$$
\int_{-(n+2) \pi}^{(n+2) \pi}|\xi|^{\alpha+\epsilon}\left|G^{(n)}(\xi)\right|^{2} d \xi \leqq C_{0} .
$$


Proof. Lemma 9 and Lemma 5 imply

$$
\int_{-\infty}^{\infty}\left|D^{k}\left(\frac{r}{n+2}\right) g^{(n)}(x)\right|^{2} d x \leqq C\left|\frac{r}{n+1}\right|^{\alpha+2 \epsilon} \leqq C_{1}\left|\frac{r}{n+2}\right|^{\alpha+2 \epsilon},
$$

for $r=1,2, \cdots, n+2$. Let $\gamma=\alpha+\epsilon<\alpha+2 \epsilon$. Let

$$
D^{k}\left(\frac{r}{n+2}\right) g^{(n)}(x)=q(x)
$$

and let $Q(\xi)$ be the Fourier transform of $q(x)$. One may easily verify that

$$
|Q(\xi)|^{2}=4^{k}\left|\sin ^{k} \frac{\xi r}{n+2}\right|\left|G^{(n)}(\xi)\right|^{2}
$$

Hence, Parseval's theorem gives

$$
\int_{-(n+2) \pi / r}^{(n+2) \pi / r}\left|\sin ^{k} \frac{\xi r}{n+2}\right|^{2}\left|G^{(n)}(\xi)\right|^{2} d \xi \leqq C_{2}\left|\frac{r}{n+2}\right|^{\alpha+2 \epsilon} .
$$

However, in the interval of integration we have

$$
\left|\sin \frac{\xi r}{n+2}\right| \geqq \frac{2}{\pi}\left|\frac{\xi r}{n+2}\right| \text {. }
$$

Hence

$$
B(r) \equiv \int_{-(n+2) \pi / r}^{(n+2) \pi / r}|\xi|^{2(k)}\left|G^{(n)}\right|^{2} d \xi \leqq C\left|\frac{n+2}{r}\right|^{2(k-\alpha-2 \epsilon)}
$$

However,

$$
\begin{aligned}
\int_{-(n+2) \pi}^{(n+2) \pi}|\xi| \gamma\left|G^{(n)}\right|^{2} d \xi \leqq & \int_{-\pi}^{\pi}|\xi| \gamma\left|G^{(n)}\right|^{2} d \xi \\
& +\sum_{r=1}^{n+1}\left|\frac{n+2}{r}\right|^{r-2(k)}[B(r)-B(r+1)] .
\end{aligned}
$$

The first term on the right hand side of (2.23) converges to

$$
\int_{-\pi}^{\pi}|\xi|^{\gamma}|G|^{2} d \xi
$$

and hence is bounded. Since $\gamma>\alpha+\epsilon$ the second term also converges as $n \rightarrow \infty$, and hence is bounded.

This result, together with Lemmas 7 and 8 give the following stronger result.

Lemma 11. Let $g(x), G(\xi), g^{(n)}(x)$ and $G^{(n)}(\xi)$ be as in the preceding lemma. 
Let $\left\{\tau_{n}(\xi)\right\}$ be any sequence of functions which converge pointwise to one and satisfy

$$
0<\rho_{0} \leqq \tau_{n}(\xi) \leqq \rho_{1}, \quad|\xi| \leqq(n+2) \pi .
$$

Let

$$
H^{(n)}(\xi)=\left\{\begin{array}{cr}
\tau_{n}(\xi) G^{(n)}(\xi), & |\xi| \leqq(n+2) \pi \\
0, & \text { otherwise }
\end{array}\right.
$$

and let

$$
R^{(n)}(\xi)=\left\{\begin{array}{cr}
\tau_{n}(\xi)|\xi|^{\alpha} G^{(n)}(\xi), & |\xi| \leqq(n+2) \pi \\
0, & \text { otherwise. }
\end{array}\right.
$$

Then, there is a subsequence $\left(n^{\prime}\right)$ such that

$$
\begin{array}{rlrl}
\left\|H^{\left(n^{\prime}\right)}(\xi)-G(\xi)\right\| & \rightarrow 0, & & \text { as } n^{\prime} \rightarrow \infty, \\
\left\|R^{\left(n^{\prime}\right)}(\xi)-|\xi|^{\alpha} G(\xi)\right\| & \rightarrow 0, & \text { as } n^{\prime} \rightarrow \infty .
\end{array}
$$

Proof. Let $\sigma_{n}(\xi)$ be the particular sequence of functions defined by

$$
\sigma_{n}(\xi)=\left\{\begin{array}{cr}
\frac{(\xi /(n+2))^{2}}{2(1-\cos \xi /(n+2))}, & |\xi| \leqq(n+2) \pi \\
0, & \text { otherwise. }
\end{array}\right.
$$

Notice that for fixed $\xi$

$$
\sigma_{n}(\xi) \rightarrow 1
$$$$
\text { as } n \rightarrow \infty
$$

and

$$
1 \leqq \sigma_{n} \leqq\left(\frac{\pi}{2}\right)^{2}, \quad|\xi| \leqq(n+2) \pi .
$$

Let $Q^{(n)}(\xi)=\sigma_{n}(\xi) H^{(n)}(\xi)$. We have

$$
\left\|Q^{(n)}-G\right\|
$$

$$
\leqq\left[\int_{|\xi| \geqq(n+2) \pi}|G|^{2} d \xi\right]^{1 / 2}+\left[\int_{|\xi| \leqslant(n+1) \pi}\left|Q^{(n)}-G\right|^{2} d \xi\right]^{1 / 2} .
$$

The first integral on the right hand side goes to zero as $n \rightarrow \infty$. Let $I$ denote the second integral on the right. Then

$$
\begin{aligned}
& I \leqq\left(\frac{\pi}{2}\right)^{2} \rho_{1}\left\{\left[\int_{|\xi| \leqq(n+2) \pi}\left|G^{(n)}(\xi)-G(\xi)\right|^{2} d \xi\right]^{1 / 2}\right. \\
& \left.+(2.28 \mathrm{~b}) \quad+\left[\int_{|\xi| \leq(n+2) \pi}\left|\frac{1}{\tau_{n} \sigma_{n}} G-G\right|^{2} d \xi\right]^{1 / 2}\right\} .
\end{aligned}
$$


The first of the integrals converges to zero because the $g^{(n)}(x)$ converge to $g(x)$ in $L_{2}$. The second converges to zero because the integrand converges to zero boundedly. A similar argument gives $\left\|H^{(n)}-G\right\| \rightarrow 0$.

The functions $G^{(n)}(\xi)$ are uniformly bounded and equicontinuous and hence there is a subsequence which satisfies the convergence hypothesis of Lemma 7. Hence the functions $1 / \tau_{n} Q^{(n)}(\xi)=\sigma_{n}(\xi) G^{(n)}(\xi)$ also contain a subsequence with those properties. Moreover,

$$
\frac{1}{2 \pi}\left\|\sigma_{n} G^{(n)}\right\|=\left[g^{(n)}, g^{(n)}\right] \rightarrow\|g\|^{2}
$$

as can easily be checked by a direct calculation. Lemma 10 and the properties of $\sigma_{n}$ and $\tau_{n}$ show that the basic estimate (2.11c) of Lemma 7 is also satisfied. Using (2.29) instead of (2.11a) we may modify the proof of Lemma 7 to obtain a subsequence $\left(n^{\prime}\right)$ so that $|\xi|^{\alpha / 2} \sigma_{n^{\prime}} G^{\left(n^{\prime}\right)}$ converges strongly to $|\xi|^{\alpha / 2} G(\xi)$. Then reversing the argument which shows that $\left\|Q^{(n)}-G\right\| \rightarrow 0$ we obtain a subsequence $\left(n^{\prime}\right)$ so that $\left\|R^{\left(n^{\prime}\right)}-|\xi|^{\alpha / 2} G\right\| \rightarrow 0$. However, every convergent subsequence must converge to the same limit. Hence

$$
\left\|R^{(n)}-|\xi|^{\alpha / 2} G\right\| \rightarrow 0 \quad \text { as } n \rightarrow \infty .
$$

LEMMA 12. Let $g(x)$ be any function vanishing outside of $[0,1]$ and satisfying

$$
g(x) \in C_{k-1}(-\infty, \infty)
$$

and $g(x)$ is infinitely differentiable except at 0,1 . Let $g^{(n)}(x)$ be the sequence of functions obtained from $g(x)$ by (2.18). Let $\tau_{n}$ be as in Lemma 11. Then

$$
(n+2)^{\alpha} \int_{-(n+2) \pi}^{(n+2) \pi}(1-\cos \xi /(n+2))^{k}\left|\tau_{n} G^{(n)}\right|^{2} d \xi \leqq M
$$

and the truncated functions

$$
R^{(n)}(\xi)=\left\{\begin{array}{cr}
(n+2)^{k}(1-\cos \xi /(n+2))^{k / 2} \tau_{n} G^{(n)}, & |\xi| \leqq(n+2) \pi \\
0, & \text { otherwise }
\end{array}\right.
$$

converge strongly to $(1 / 2)^{k / 2}|\xi|^{k} G(\xi)$.

Proof. Since $g(x)$ has $L_{2}$ derivatives of order $k$

$$
(n+2)^{\alpha} \int_{-\infty}^{\infty}\left|D^{k}\left(\frac{1}{n+2}\right) g(x)\right|^{2} d x \leqq M .
$$

Let $q(x)=(n+2)^{k} D^{k}(1 /(n+2)) g(x)$ and let $Q(\xi)$ be its Fourier transform. Then

$$
|Q(\xi)|^{2}=2^{\alpha}(n+2)^{\alpha}|1-\cos \xi /(n+2)|^{k}\left|G^{(n)}\right|^{2} .
$$

Hence (2.31) follows from Parseval's theorem. 
It is an elementary exercise to verify that

$$
\int_{-\infty}^{\infty}\left|(n+2)^{k} D^{k} g^{n}(x)-\left(\frac{d}{d x}\right)^{k} g(x)\right|^{2} d x \rightarrow 0, \quad \text { as } n \rightarrow \infty .
$$

Hence $2^{k}(n+2)^{k}(1-\cos \xi /(n+2))^{k / 2} G^{(n)}(\xi)$ converges strongly to $|\xi|^{k / 2} G(\xi)$. We now repeat the argument of the first part of Lemma 11 to handle the factor $\tau_{n}$.

3. The proof of the theorem. Remembering that $x_{j}=j /(n+2)$ we may express the results of Lemma 1 as

Lemma 13. Let $h(x), g(x) \in P_{n}$ and let $H(\xi)$ and $G(\xi)$ be their Fourier transforms. Let $H=\left(h_{j}\right)$ and $G=\left(g_{j}\right)$ be the associated $(n+1)$ vectors. Then

$$
\begin{aligned}
& (n+2)^{\alpha}\left(H, T_{n} G\right)=(n+2)\left[h, S_{n} g\right] \\
& {\left[h, S_{n} g\right]=\frac{(n+2)^{\alpha}}{2 \pi} \int_{-(n+2) \pi}^{(n+2) \pi}\left[\sigma_{n}(\xi)\right]^{2} f(\xi /(n+2)) H(\xi) \bar{G}(\xi) d \xi}
\end{aligned}
$$

and

$$
[h, g]=\frac{1}{n+2}(H, G)=\frac{1}{2 \pi} \int_{-(n+2) \pi}^{(n+2) \pi}\left[\sigma_{n}(\xi)\right]^{2} H(\xi) \bar{G}(\xi) d \xi,
$$

where $\left\{\sigma_{n}(\xi)\right\}$ is the sequence of functions defined by (2.27a).

Proof. Direct computation.

With this formula, and the results of the preceding section, one may easily establish a bound for the quantities $(n+2)^{\alpha} \lambda_{\nu, n}$.

Lemma 14. For every $\alpha>0$ and every $\nu \geqq 1$ there is a constant $M_{\nu}$, depending only on $\alpha$ and $\nu$, such that

$$
(n+2)^{\alpha} \lambda_{\nu, n} \leqq M_{v}
$$

And, in fact,

$$
\operatorname{Lim} \operatorname{Sup}(n+2)^{\alpha} \lambda_{\nu, n} \leqq\left[\sigma^{2} /(2 k) !\right]^{\alpha / 2 k} \Lambda_{\nu} .
$$

Proof. Let $g(x)$ be any function which is a linear combination of the $\phi^{(j)}(x), j=1,2, \cdots, \nu$, the first $\nu$ eigenfunctions of $k_{0}(x, y)$. Let $\left\{g^{(n)}(x)\right\}$ be the functions obtained from $g(x)$ by (2.18) and let $\left\{G^{(n)}(\xi)\right\}$ be their Fourier transforms. Let $\left\{\phi^{n, j}(x)\right\}$ be the functions obtained from the $\phi^{(j)}(x)$. For $n \gg \nu$ one can easily verify that the $\phi^{n, j}(x)$ are linearly independent, and. hence so are their associated $n+1$ vectors. From Lemma 3 we have

$$
(n+2)^{\alpha} \lambda_{\nu, n} \leqq \operatorname{Max}_{0} \frac{\left[g^{(n)}, S_{n} g^{(n)}\right]}{\left[g^{(n)}, g^{(n)}\right]} .
$$

Let us normalize $g^{(n)}$ so that $\left[g^{(n)}, g^{(n)}\right]=1$. If $\alpha<2 k(\alpha)$ we have 


$$
(n+2)^{\alpha} \lambda_{\nu, n} \leqq \operatorname{Max}_{0} \frac{1}{2 \pi} \int_{-(n+2) \pi}^{(n+2) \pi} \beta|\xi|^{\alpha}(1+r(\xi /(n+2)))\left[\sigma_{n}(\xi)\right]^{2}\left|G^{(n)}\right|^{2} d \xi
$$

and (3.2) follows from Lemma 10. Moreover, (3.2a) follows from Lemma 11. If $\alpha=2 k(\alpha)$ we have

$$
(n+2)^{\alpha} \lambda_{\nu, n} \leqq \operatorname{Max}_{0} \frac{(n+2)^{\alpha}}{2 \pi} \int_{-(n+2) \pi}^{(n+2) \pi} \beta(1-\cos \xi /(n+2))^{k}\left|\sigma_{n}\right|^{2}\left|G^{(n)}\right|^{2} d \xi
$$

Thus (3.2) and (3.2a) follow from Lemma 12.

LEMmA 15. Let $\left\{h^{(n)}(x)\right\} \in P_{n}$ be the eigenfunctions of $S_{n}$ associated with $(n+2)^{\alpha} \lambda_{v, n}$ normalized so that $[h, h]=1$. Lemma 14 enables one to select a subsequence $\left\{h^{\left(n^{\prime}\right)}(x)\right\}$ so that the quantities $\left(n^{\prime}+2\right)^{\alpha} \lambda_{\nu, n^{\prime}}$ converge to a finite number, say $\Lambda_{\nu}^{0}$. Let $\left\{h^{\left(n^{\prime}\right)}(x)\right\}$ be any such sequence. Then

$$
\Lambda_{\nu}^{0}=\left[\frac{\sigma^{2}}{(2 k) !}\right]^{2 k / \alpha} \Lambda_{j}
$$

for some $j$. Moreover, there is a sub-subsequence, which we again call $h^{(n)}(x)$, such that the truncated Fourier transforms converge strongly to $\Phi^{(j)}(\xi)$. That is, let

$$
Q_{n^{\prime}}(\xi)=\left\{\begin{array}{cl}
H^{\left(n^{\prime}\right)}(\xi), & |\xi| \leqq\left(n^{\prime}+2\right) \pi \\
0, & \text { otherwise. }
\end{array}\right.
$$

Then

$$
\left\|Q^{(n)}-\Phi^{(j)}\right\| \rightarrow 0
$$

where $\Phi^{(j)}(\xi)$ is the Fourier transform of an eigenfunction $\phi^{(j)}(x)$ associated with $\Lambda_{j}$.

Proof. Since $\left[h^{\left(n^{\prime}\right)}, h^{\left(n^{\prime}\right)}\right]=1$ the functions $Q_{n^{\prime}}(\xi)$ are uniformly bounded and equicontinuous. Moreover, using Lemma 13 and $(2.27 \mathrm{c})$ we have

$$
\left\|Q^{\left(n^{\prime}\right)}\right\| \geqq\left(\frac{2}{\pi}\right)^{2}
$$

Thus we may pick out a subsequence, which we call $Q^{(n)}$, which converges weakly in $L_{2}(-\infty, \infty)$, converges pointwise, and converges uniformly on every finite interval. Just as in Lemma 7 we have

$$
\int_{-a}^{a}\left|Q^{(n)}\right|^{2} d \xi \geqq\left(\frac{2}{\pi}\right)^{2}-\frac{M}{a^{\alpha}} .
$$

Thus we may conclude: the subsequence $Q^{(n)}(\xi)$ converges weakly and pointwise to a nonzero function $Q(\xi)$.

Let $g(x)=\phi^{(j)}(x)$ and let $\left\{g^{(n)}(x)\right\}$ be the sequence obtained from $\phi^{(j)}$ by 
(2.18). Let $G^{(n)}(\xi)$ be the Fourier transform of $g^{(n)}(x)$.

For every $j$ we have

$$
\left[g^{(n)}, S_{n} h^{(n)}\right]=(n+2)^{\alpha} \lambda_{\nu, n}\left[g^{(n)}, h^{(n)}\right] .
$$

Let $\alpha<2 k(\alpha)$. Then (3.7) becomes

$$
\begin{array}{r}
\int_{-(n+2) \pi}^{(n+2) \pi} \beta\left\{|\xi| \alpha / 2[1+r(\xi /(n+2))]\left|\sigma_{n}\right|^{2} G^{(n)}\right\}\left\{|\xi| \alpha / 2 \bar{Q}^{(n)}(\xi)\right\} d \xi \\
=(n+2)^{\alpha} \lambda_{\nu, n} \int_{-(n+2) \pi}^{(n+2) \pi}\left|\sigma_{n}\right|{ }^{2} G^{(n)} \bar{Q}^{(n)} d \xi
\end{array}
$$

The basic estimate (3.2) allows us to select a sub-subsequence for which the functions $|\xi|^{\alpha / 2} Q^{(n)}(\xi)$ also converge weakly, and hence pointwise, etc. Lemma 11 assures us that the functions $|\xi|^{\alpha / 2}[1+r(\xi /(n+2))]\left|\sigma_{n}\right|^{2} G^{(n)}(\xi)$ converge strongly to $|\xi|^{\alpha / 2} \Phi^{(j)}(\xi)$. Hence we find

$$
\int_{-\infty}^{\infty} \beta|\xi|^{\alpha} \Phi^{(j)}(\xi) \bar{Q}(\xi) d \xi=\Lambda_{\nu}^{0} \int_{-\infty}^{\infty} \Phi^{(j)}(\xi) \bar{Q}(\xi) d \xi
$$

Thus, Lemma 4 implies that either $\Lambda_{\nu}^{0}=\beta \Lambda_{j}$ for some $j$ or $Q(\xi) \equiv 0$. But $Q(\xi) \not \equiv 0$. Thus, in this case, (3.4) is verified. Looking at (3.8) once more we see that $Q(\xi)=\Phi^{(j)}(\xi)$. Finally

$$
\left[h^{(n)}, S_{n} h^{(n)}\right]=(n+2)^{\alpha} \lambda_{\nu, n} \rightarrow \Lambda_{\nu}^{0}
$$

and (3.4) implies that $\left\||\xi|^{\alpha / 2} Q^{(n)}\right\| \rightarrow\left\||\xi|^{\alpha / 2} Q\right\|$ which implies the strong convergence of $|\xi|^{\alpha / 2} Q^{(n)}$ and a fortiori the strong convergence of $Q^{(n)}$.

Now let $\alpha=2 k(\alpha)$. Then (3.7) becomes

$$
\begin{aligned}
(n+2)^{\alpha} \int_{-(n+2) \pi}^{(n+2) \pi} \beta\left\{(1-\cos \xi /(n+2))^{k / 2}\left|\sigma_{n}\right|{ }^{2} G^{(n)}\right\} \\
\cdot\left\{(1-\cos \xi /(n+2))^{k / 2} \bar{Q}^{(n)}(\xi)\right\} d \xi \\
=(n+2)^{\alpha} \lambda_{\nu, n} \int_{-(n+2) \pi}^{(n+2) \pi}\left|\sigma_{n}\right|{ }^{2} G^{(n)} \bar{Q}^{(n)} d \xi .
\end{aligned}
$$

The desired conclusion now follows precisely as above, making use of Lemma 12 rather than Lemma 11.

Our proof is almost complete. We must only show

$$
\Lambda_{\nu}^{0}=\left(\frac{\sigma^{2}}{(2 k) !}\right)^{\alpha / 2 k} \Lambda_{\nu} .
$$

For $\nu=1$ this follows from Lemma 14 and the chosen ordering of the $\Lambda_{\nu}$. The general case follows by induction. It is only necessary to use the orthog- 
onality properties of the eigenfunctions $\left\{h^{(n)}(x)\right\},\left\{\phi^{(i)}\right\}$. A more thorough argument may be found in [10].

\section{REFERENCES}

1. R. Courant and D. Hilbert, Methods of mathematical physics, vol. 1 (English translation), New York, Interscience, 1953.

2. U. Grenander and G. Szegö, Toeplitz forms and their applications, Berkeley, University of California Press, 1958.

3. M. Kac, W. L. Murdock and G. Szegö, On the eigenvalues of certain Hermitian forms, J. Rational Mech. Anal. vol. 2 (1953) pp. 767-800.

4. S. V. Parter, Extreme eigenvalues of Toeplitz forms and applications to elliptic difference equations, Trans. Amer. Math. Soc. vol. 99 (1961) pp. 153-193.

5. - On the extreme eigenvalues of truncated Toeplitz matrices, Bull. Amer. Math. Soc. vol. 67 (1961) pp. 191-196.

6. F. Riesz and B. Nagy, Functional analysis, New York, F. Unger Publishing Co., 1955.

7. E. C. Titchmarsh, Theory of fourier integrals, Oxford, Clarendon Press, 1937.

8. H. Widom, On the eigenvalues of certain Hermitian operators, Trans. Amer. Math. Soc. vol. 88 (1958) pp. 491-522.

9. - Stable processes and integral equations, Trans. Amer. Math. Soc. vol. 98 (1961) pp. $430-449$.

10. —, Extreme eigenvalues of translation kernels, Trans. Amer. Math. Soc. vol. 99 (1961) pp. 252-262.

Cornell University, ITHACA, New York 